\title{
DETERMINAÇÃO DAS ISOTERMAS DE EQUILÍBRIO HIGROSCÓPICO DE FRUTOS DE CRAMBE PELO MÉTODO DINÂMICO
}

\author{
DETERMINATION OF HYGROSCOPIC EQUILIBRIUM ISOTHERMS OF CRAMBE \\ FRUIT BY DYNAMIC METHOD
}

\author{
Lílian Moreira COSTA ${ }^{1}$; Osvaldo RESENDE ${ }^{\mathbf{1}}$; Daniel Emanuel Cabral de OLIVEIRA ${ }^{1}$ \\ 1. Instituto Federal Goiano, Rio Verde, GO, Brasil. Imctpg@yahoo.com.br
}

\begin{abstract}
RESUMO: Objetivou-se neste trabalho determinar as isotermas de sorção dos frutos de crambe pelo método dinâmico para diversas condições de temperatura e atividades de água e ajustar diferentes modelos matemáticos aos dados experimentais, bem como obter os valores do calor isostérico de dessorção em função do teor de água de equilíbrio do produto. $\mathrm{O}$ teor de água de equilíbrio dos frutos de crambe foi determinado pelo método dinâmico-gravimétrico para temperaturas de $25,30,35,40$ e $45^{\circ} \mathrm{C}$ e atividades de água para cada temperatura, entre 0,270 a 0,825 . A partir dos resultados obtidos calculou-se o calor isostérico para cada teor de água de equilíbrio. Aos dados experimentais foram ajustados diversos modelos matemáticos disponíveis na literatura (Chung-pfost, Copace, GAB, Halsey Modificado, Sabbah, Sigma Copace, Cavalcanti Mata, Henderson Modificado, Henderson, BET, Oswin e GAB mod). Observou-se que o teor de água de equilíbrio decresce com o aumento da temperatura para uma dada atividade de água à semelhança dos produtos higroscópicos. O modelo de Sigma Copace foi o que descreveu melhor a higroscopicidade dos frutos de crambe. Observou-se que o calor isostérico aumenta com a diminuição do teor de água do produto, indicando aumento da energia necessária para a remoção de água. Os valores de calor isostérico para os frutos de crambe na faixa de teor de água de 3,85 a 11,86 (\% b.s.) variaram de 4731,73 a $2698,35 \mathrm{~kJ} \mathrm{~kg}^{-1}$.
\end{abstract}

PALAVRAS-CHAVE: Crambe abyssinica. Oleaginosa. Modelagem matemática. Calor isostérico.

\section{INTRODUÇÃO}

A criação de fontes de energias renováveis vem se sobressaindo no quadro mundial, dentre elas o biodiesel que agrega e incentiva o desenvolvimento de diversas culturas com potencial para a produção (MELLO, 2011).

A cultura do crambe (Crambe abyssinica Hochst) vem ganhando importância no Brasil devido a sua aptidão para a produção de biodiesel e potencial para semeadura entre os meses de abril e maio, caracterizando safrinha na região centrooeste. Originário da região quente e seca da Etiópia foi domesticado na região fria e seca do Mediterrâneo (KNIGHTS, 2008; OPLINGER et al., 2008). A utilização do crambe para fins industriais como visto anteriormente vem aumentando a cada ano. No entanto, para que se atenda a demanda é necessário o desenvolvimento de técnicas de manejo que possam vir a agregar uma maior produtividade.

Neste contexto, dentre as etapas póscolheita de destaque tem-se o armazenamento dos frutos. Durante esta fase podem ocorrer mudanças físicas, químicas e microbiológicas que, dependendo da interação entre estes fatores e o ambiente, podem ocasionar perdas na sua qualidade. Assim, faz-se necessário o conhecimento das relações existentes entre o produto, a temperatura e a umidade relativa do ar, objetivando iniciativas e estudos com a finalidade de amenizar estas possíveis alterações (RESENDE et al., 2006) .

Para determinar as isotermas de sorção pode-se utilizar o método gravimétrico ou $o$ higrométrico. No método higrométrico, o teor de água do material é mantido constante até que o ar circundante atinja o valor constante de equilíbrio, sendo a atividade de água medida via higrômetro ou manômetro. $\mathrm{O}$ método gravimétrico, a temperatura do ar e a atividade de água são mantidas constantes até que o teor de água da amostra atinja o valor de equilíbrio, sendo que o ar pode ser circulado (método dinâmico) ou estagnado (método estático), desta forma o tempo para o produto entrar em equilíbrio é menor no método dinâmico (MOREIRA, 2000).

A utilização de equações matemáticas para estimar o teor de água de equilíbrio higroscópico apresenta a vantagem de predição de valores de atividade de água do produto, em condições ambientais de difícil determinação experimental. A relação entre o teor de água de um produto e a umidade relativa de equilíbrio, a uma determinada temperatura, pode ser expressa por meio de curvas características de teor de água de equilíbrio, denominadas isotermas. Numerosos são os modelos com capacidade de predizer uma isoterma (RESENDE et al., 2006).

Para se reduzir ao mínimo o processo de deterioração, as sementes devem ser armazenadas 
Determinação das isotermas...

adequadamente, garantindo assim a manutenção de estoque regulador para os anos subsequentes de baixa produção. Como todo material higroscópico, as sementes cedem ou absorvem água do ar que as envolve; assim, se a pressão de vapor de água na semente for menor do que a do ar ocorre a absorção de umidade (sorção) e, no caso inverso, a semente cede água para o ar (dessorção). Quando a pressão de vapor de água da superfície da semente se iguala à pressão do ar ambiente, obtém-se o teor de água de equilíbrio (NELLIST; HUGUES, 1973). Por serem altamente higroscópicas, as sementes têm comportamento diferenciado nas isotermas de sorção. Sementes ricas em óleo apresentam teores de água de equilíbrio mais baixos em relação às sementes amiláceas, quando armazenadas em condições ambientais semelhantes, pois absorvem menos água, por serem hidrófobas (BROOKER et al., 1992).

Segundo Sopade e Ajisegiri (1994) o conhecimento do calor isostérico em função do teor de água de equilíbrio é essencial nos estudos de secagem e armazenamento de produtos agrícolas, servindo para estimar as necessidades energéticas do processo de secagem fornecendo, também, dados sobre o estado da água no produto.

Diante da importância do conhecimento da higroscopicidade dos produtos agrícolas, objetivouse neste trabalho determinar as isotermas de sorção dos frutos de crambe pelo método dinâmico para diversas condições de temperatura e atividades de água e ajustar diferentes modelos matemáticos aos dados experimentais, bem como obter os valores do calor isostérico de dessorção em função do teor de água de equilíbrio do produto.

\section{MATERIAIS E MÉTODOS}

Este trabalho foi desenvolvido no Laboratório de Pós-Colheita de Produtos Vegetais
COSTA, L. M.; RESENDE, O.; OLIVEIRA, D. E. C.

do Instituto Federal de Educação, Ciência e Tecnologia Goiano - IF Goiano - Campus Rio Verde, localizado no município de Rio Verde, GO. Para a condução do experimento, foram utilizados frutos de crambe (Crambe abyssinica) cultivados na área experimental do IF Goiano - Câmpus Rio Verde.

Os frutos de crambe foram colhidos manualmente com teor de água de, aproximadamente, 26,0 (\% b.s.). Os teores de água dos frutos de crambe foram determinados por gravimetria, utilizando-se a estufa a $105 \pm 3{ }^{\circ} \mathrm{C}$, durante 24 horas (BRASIL, 2009).

Para obtenção do teor de água de equilíbrio higroscópico dos frutos de crambe foi utilizado o método dinâmico-gravimétrico. A dessorção do produto em camada delgada foi realizada para diferentes condições controladas de temperatura (25, $30,35,40$ e $45^{\circ} \mathrm{C}$ ) e atividades de água entre 0,27 e 0,82 , até que o produto atingisse seu teor de água de equilíbrio com a condição do ar especificada.

As condições ambientais para realização dos testes de higroscopicidade foram fornecidas por meio de uma câmara condicionadora de atmosfera. As amostras, contendo cada uma $10 \mathrm{~g}$ de produto, foram envolvidas por um tecido permeável (voile) para permitir a passagem do ar através do produto e colocadas no interior do equipamento.

Durante o processo as amostras foram pesadas periodicamente e o equilíbrio higroscópio foi atingido quando a variação da massa permaneceu, aproximadamente, invariável durante três pesagens consecutivas.

Aos dados experimentais do teor de água de equilíbrio foram ajustados os modelos matemáticos frequentemente utilizados para representação da higroscopicidade de produtos agrícolas, cujas expressões estão apresentadas na Tabela 1.

Tabela 1. Modelos matemáticos utilizados para predizer o fenômeno de higroscopicidade de produtos agrícolas.

$\begin{array}{cc}\text { Designação do modelo } & \text { Modelo } \\ \mathrm{Xe}^{*}=\mathrm{a}-\mathrm{b} \cdot \operatorname{In}\left[-(\mathrm{T}+\mathrm{c}) \cdot \operatorname{In}\left(\mathrm{a}_{\mathrm{w}}\right)\right] & \text { Chung-pfost } \\ \mathrm{Xe}{ }^{*}=\exp \left[\mathrm{a}-(\mathrm{b} \cdot \mathrm{T})+\left(\mathrm{c} \cdot \mathrm{a}_{\mathrm{w}}\right)\right] & \text { Copace } \\ \mathrm{Xe}{ }^{*}=\left(\mathrm{a} \cdot \mathrm{b} \cdot \mathrm{c} \cdot \mathrm{a}_{\mathrm{w}}\right) /\left[\left(1-\mathrm{c} \cdot \mathrm{a}_{\mathrm{w}}\right) \cdot\left(1-\mathrm{c} \cdot \mathrm{a}_{\mathrm{w}}+\mathrm{b} \cdot \mathrm{c} \cdot \mathrm{a}_{\mathrm{w}}\right)\right] & \text { GAB } \\ \mathrm{Xe}^{*}=\left[\exp (\mathrm{a}-\mathrm{b} \cdot \mathrm{T}) /-\operatorname{In}\left(\mathrm{a}_{\mathrm{w}}\right)\right]^{1 / \mathrm{c}} & \text { Halsey Modificado }\end{array}$




$$
\begin{aligned}
& \mathrm{Xe}^{*}=\mathrm{a} \cdot\left(\mathrm{a}_{\mathrm{w}} \mathrm{b} / \mathrm{T}^{\mathrm{c}}\right) \\
& X e^{*}=\exp \left\{a-(b \cdot T)+\left[c \cdot \exp \left(a_{w}\right)\right]\right\} \\
& \mathrm{Xe} e^{*}=\left[\log \left(1-\mathrm{a}_{\mathrm{w}}\right) /\left(\mathrm{a} \cdot\left(\mathrm{T}^{\mathrm{b}}\right)\right)\right]^{\frac{1}{\mathrm{c}}} \\
& \mathrm{Xe}^{*}=\{\ln (1-\mathrm{UR}) /[-\mathrm{a} \times(\mathrm{T}+\mathrm{b})]\}^{1 / \mathrm{c}} \\
& X e^{*}=\left[\operatorname{In}\left(1-a_{w}\right) /(-\mathrm{a} \cdot \mathrm{T}+273,16)\right]^{1 / \mathrm{c}} \\
& \mathrm{Xe}^{*}=\left\{1 /\left[\left(1-\mathrm{a}_{\mathrm{w}}\right) \cdot(1 / \mathrm{a} \cdot \mathrm{b}+((\mathrm{a}-1) / \mathrm{a} \cdot \mathrm{b}))\right]\right\} \\
& \mathrm{Xe}^{*}=(\mathrm{a}+\mathrm{bT}) /\left[\left(1-\mathrm{a}_{\mathrm{w}}\right) / \mathrm{a}_{\mathrm{w}}\right]^{1 / \mathrm{c}} \\
& \text { Sabbah } \\
& \text { Sigma Copace } \\
& \text { Cavalcanti Mata } \\
& \text { Henderson Modificado } \\
& \text { Henderson } \\
& \text { BET } \\
& \text { Oswin } \\
& X e^{*}=\left(a \cdot b \cdot a_{w}\right) \cdot \frac{\left(\frac{c}{T}\right)}{\left(1-b \cdot a_{w}+\left(\frac{c}{T}\right) \cdot b \cdot a_{w}\right) \cdot\left(1-b \cdot a_{w}\right)} \\
& \mathrm{Xe}^{*} \text { : teor de água de equilíbrio, \% b.s. } \\
& \mathrm{a}_{\mathrm{W}}: \text { atividade de água, decimal } \\
& \mathrm{T} \text { : temperatura, }{ }^{\circ} \mathrm{C}
\end{aligned}
$$

Para o ajuste dos modelos matemáticos foi realizada a análise de regressão não linear, pelo método Gauss Newton, utilizando-se um programa estatístico. Para verificar o grau de ajuste de cada modelo foi considerado a significância do coeficiente de regressão pelo teste $\mathrm{t}$, adotando o nível de 5\% de significância, a magnitude do coeficiente de determinação $\left(\mathrm{R}^{2}\right)$, os valores do erro médio relativo $(\mathrm{P})$, do erro médio estimado (SE), o

$$
\begin{gathered}
P=\frac{100}{n} \sum \frac{|Y-\hat{Y}|}{Y} \\
S E=\sqrt{\frac{\sum(Y-\hat{Y})^{2}}{G L R}} \\
\chi^{2}=\sum \frac{(\mathrm{Y}-\hat{\mathrm{Y}})^{2}}{\mathrm{GLR}}
\end{gathered}
$$

teste de Qui-quadrado $\left(\chi^{2}\right)$ ao nível de significância de $1 \%$ e o intervalo de confiança a $99 \%(\mathrm{P}<0,01)$. Considerou-se o valor do erro médio relativo inferior a $10 \%$ como um dos critérios para a seleção dos modelos de acordo com Mohapatra e Rao (2005). Os erros médios relativo e estimado, e o teste de Qui-quadrado para cada um dos modelos, foram calculados conforme as seguintes expressões, respectivamente:

em que:

Y : valor experimental

$\hat{\mathrm{Y}}$. valor estimado pelo modelo

$\mathrm{N}$ : número de observações experimentais

GLR : graus de liberdade do modelo (número de observações menos o número de parâmetros do modelo) 
Os valores do calor isostérico líquido de sorção (ou entalpia diferencial), para cada teor de

equação Clausius-Clayperon

(IGLESIAS; CHIRIFE, 1976) como mostrada a seguir: água de equilíbrio, foram obtidos por meio da

em que:

$\mathrm{a}_{\mathrm{w}}$ : atividade de água, decimal

$\mathrm{T}_{\mathrm{a}}$ : temperatura absoluta, $\mathrm{K}$

$\Delta \mathrm{h}_{\mathrm{st}}$ : calor isostérico líquido de sorção, $\mathrm{kJ} \mathrm{kg}^{-1}$

$\mathrm{R}$ : constante universal dos gases, $8,314 \mathrm{~kJ} \mathrm{kmol}^{-1} \mathrm{~K}^{-1}$, sendo para o vapor d'água $0,4619 \mathrm{~kJ} \mathrm{~kg}^{-1} \mathrm{~K}^{-1}$

Integrando a Equação 16 e assumindo que o calor isostérico líquido de sorção é independente da temperatura, obtém-se o calor isostérico líquido de sorção, para cada teor de água de equilíbrio, conforme a Equação 17 (WANG; BRENNAN, 1991):

$$
\operatorname{In}\left(\mathrm{a}_{\mathrm{w}}\right)=-\left(\frac{\Delta \mathrm{h}_{\mathrm{st}}}{\mathrm{R}}\right) \cdot \frac{1}{\mathrm{~T}_{\mathrm{a}}}+\mathrm{C}
$$

em que:

C: coeficiente do modelo

Os valores de atividade de água, temperatura e teor de água de equilíbrio foram obtidos a partir das isotermas de dessorção frutos de crambe, utilizando o modelo de melhor ajuste aos dados experimentais. $\mathrm{O}$ calor isostérico integral de

$$
\mathrm{Q}_{\mathrm{st}}=\Delta \mathrm{h}_{\mathrm{st}}+\mathrm{L}=\mathrm{a} \cdot \exp \left(-\mathrm{b} \cdot \mathrm{Xe} \mathrm{e}^{*}\right)+\mathrm{L}
$$

em que,

$\mathrm{Q}_{\mathrm{st}}: \quad$ calor isostérico integral de sorção, $\mathrm{kJ}$ kg-1

$\mathrm{L}: \quad$ calor latente de vaporização da água livre, $\mathrm{kJ}$ kg-1

$\mathrm{Xe}^{*}$ : teor de água de equilíbrio, \% b.s.

a, b: coeficientes do modelo

O calor latente de vaporização da água livre (L), em $\mathrm{kJ} \mathrm{kg}^{-1}$, necessário ao cálculo de $\mathrm{Q}_{\mathrm{st}}$, foi obtido utilizando-se a temperatura média (T) na

$$
\mathrm{L}=2502,2-2,39 . \mathrm{T}
$$

\section{RESULTADOS E DISCUSSÕES}

Na Tabela 2 estão apresentados os valores médios do teor de água de equilíbrio higroscópico dos frutos de crambe obtidos por dessorção para as temperaturas de $25,30,35,40$ e $45{ }^{\circ} \mathrm{C}$ e atividade de água entre 0,270 a 0,825 (decimal). Verifica-se que para uma mesma temperatura, com a variação da atividade de água, tem-se um aumento sensível do teor de água de equilíbrio. Além disso, o teor de água de equilíbrio dos frutos de crambe aumenta com a diminuição da temperatura.

O mesmo fenômeno foi constatado por Hofsky et al. (2007) e Sousa et al. (2013), ao estudarem a higroscopicidade de pitanga em pó e nabo forrageiro, verificaram que o teor de água de equilíbrio decresce com o aumento de temperatura, para uma mesma umidade relativa, seguindo a sorção foi obtido adicionando-se aos valores de calor isostérico líquido de sorção, o valor do calor latente de vaporização da água livre de acordo com a equação 18: faixa em estudo, em ${ }^{\circ} \mathrm{C}$, por meio da seguinte equação:

tendência da maioria dos produtos vegetais já estudados.

De acordo com Campos et al. (2009) a diminuição do teor de água de equilíbrio com o aumento da temperatura pode ser justificada com base no aumento da pressão de vapor da água no ar e na superfície do produto. Os autores relatam ainda que este aumento é maior na superfície do produto visto que a mesma apresenta maior número de moléculas de água que o ar. Maior pressão de vapor implica em maior perda de água, para que se atinja o equilíbrio (FERREIRA; PENA, 2003)

Os parâmetros dos modelos ajustados aos valores de teor de água de equilíbrio higroscópico para os frutos de crambe, obtidos por dessorção, pelo método dinâmico para diferentes condições de temperatura e atividades de água, estão apresentados na Tabela 3. 
Tabela 2. Valores médios do teor de água de equilíbrio (\% b.s.) dos frutos de crambe, obtidos pelo método dinâmico e por dessorção, em função da temperatura $\left({ }^{\circ} \mathrm{C}\right)$ e da atividade de água (decimal, $\left.\pm 0,03\right)$.

\begin{tabular}{cccccc}
\hline $\begin{array}{c}\text { Atividade de água } \\
(\text { decimal })\end{array}$ & 25 & 30 & 35 & 40 & 45 \\
\cline { 2 - 6 } 0,270 & - & - & - & 3,86 & - \\
0,275 & - & - & - & - & 3,97 \\
0,355 & - & - & - & - & 4,13 \\
0,375 & - & 4,85 & 4,64 & - & - \\
0,405 & - & - & - & 4,26 & - \\
0,450 & 6,13 & - & - & 4,89 & - \\
0,525 & 7,39 & 6,24 & 6,21 & 5,53 & - \\
0,600 & 7,72 & - & - & - & - \\
0,625 & - & 7,69 & - & - & - \\
0,655 & - & - & - & - & 7,02 \\
0,675 & - & - & 8,04 & - & - \\
0,700 & - & 8,38 & - & - & - \\
0,775 & - & 9,74 & - & - & - \\
0,825 & - & - & 10,86 & - & - \\
\hline
\end{tabular}

Tabela 3. Modelos ajustados aos teores de água de equilíbrio higroscópico para os frutos de crambe, com seus respectivos coeficientes de determinação $\left(\mathrm{R}^{2}\right)$, erros médios relativo $(\mathrm{P})$, erros médios estimado $(\mathrm{SE})$ e Qui-quadrado $\left(\chi^{2}\right)$.

\begin{tabular}{|c|c|c|c|c|}
\hline \multirow{2}{*}{ Modelos } & $\mathrm{R}^{2}$ & $\mathrm{P}$ & \multirow{2}{*}{$\begin{array}{c}\text { SE } \\
\text { decimal }\end{array}$} & \multirow{2}{*}{$\begin{array}{c}\chi^{2} \\
\text { decimal } \\
\times 10^{-10}\end{array}$} \\
\hline & \multicolumn{2}{|c|}{$\%$} & & \\
\hline Chung-pfost & 97,47 & 4,546 & 0,340 & 0,116 \\
\hline Copace & 98,03 & 3,655 & 0,300 & 0,090 \\
\hline GAB & 93,79 & 6,212 & 0,533 & 0,284 \\
\hline Halsey Modificado & 97,85 & 3,982 & 0,314 & 0,098 \\
\hline Sabbah & 95,18 & 5,915 & 0,470 & 0,221 \\
\hline Sigma Copace & 98,18 & 3,534 & 0,289 & 0,083 \\
\hline Cavalcanti Mata & 97,78 & 4,012 & 0,319 & 0,102 \\
\hline Henderson Modificado & 97,73 & 4,082 & 0,322 & 0,104 \\
\hline Henderson & 95,23 & 5,785 & 0,454 & 0,206 \\
\hline BET & 62,14 & 13,297 & 1,277 & 1,632 \\
\hline Oswin & 98,10 & 3,887 & 0,295 & 0,087 \\
\hline GAB mod & 96,94 & 4,913 & 0,374 & 0,140 \\
\hline
\end{tabular}

Verifica-se que de um modo geral, os modelos apresentaram elevados valores do coeficiente de determinação, superior a $93 \%$, exceto o modelo de BET que apresentou 62,14\%. Para uma análise aprimorada, utilizaram-se outros parâmetros estatísticos para respaldar a seleção do melhor modelo, segundo Madamba et al. (1996), a utilização do coeficiente de determinação como o único critério de avaliação para a seleção dos modelos não lineares não constitui um bom parâmetro para representação o fenômeno. Nota-se que o valor do erro médio relativo $(\mathrm{P})$ foi superior a $10 \%$ somente para o modelo de BET e inferior a $10 \%$ para os demais modelos testados, que de acordo com Mohapatra e Rao (2005), indica uma representação adequada do fenômeno estudado.

Quanto aos valores obtidos referentes ao erro médio estimado (SE), nota-se que o modelo de 
BET foi o que apresentou o maior valor quando comparados aos demais. Em relação ao teste de Quiquadrado $\left(\chi^{2}\right)$, os doze modelos analisados se encontram no intervalo de confiança de $95 \%$. Entretanto, comparando a magnitude dos valores, somente o modelo de BET apresentou maior valor em comparação aos demais.

Desta forma, todos os modelos testados ajustaram-se satisfatoriamente aos teores de água de equilíbrio higroscópico para os frutos de crambe, com exceção ao modelo de BET que, nas condições que foi realizado este trabalho, não se ajustou adequadamente. Portanto, dentre os modelos analisados, o modelo Sigma Copace foi selecionado por apresentar o maior coeficiente de determinação, e os menores valores de erros médios relativo e estimado e do teste de Qui-quadrado.

Mesquita et al. (2001), também obtiveram melhor ajuste do modelo de Sigma Copace para as
COSTA, L. M.; RESENDE, O.; OLIVEIRA, D. E. C.

espécies de jacarandá-da-bahia (Dalbergia nigra Fr. All.) e angico-vermelho (Anadenanthera macrocarpa Benth.), em função de duas temperaturas, 5 e $25{ }^{\circ} \mathrm{C}$ e umidade relativa entre 32,5 a $98,5 \%$. Sousa et al. (2013) avaliando dois métodos de determinação do teor de água de equilíbrio higroscópico, dinâmico e estático, conseguiram o melhor ajuste para o método estático os modelos de Sigma Copace, Oswin, Halsey Modificado e Copace nas sementes de nabo forrageiro para as temperaturas de $25,30,35$ e 40 ${ }^{\circ} \mathrm{C}$.

Verifica-se na Tabela 4, que o modelo matemático de Sigma Copace utilizado para descrever a higroscopicidade dos frutos de crambe apresentou, para os seus coeficientes, significância ao nível de $1 \%$ pelo teste $t$.

Tabela 4. Coeficientes do modelo de Sigma Copace ajustados aos teores de água de equilíbrio higroscópico para os frutos de crambe obtidos pelo método dinâmico.

\begin{tabular}{cccc}
\hline Equação & \multicolumn{3}{c}{ Coeficientes } \\
\cline { 2 - 4 } & $\mathrm{a}$ & $\mathrm{b}$ & $\mathrm{c}$ \\
\hline $\mathrm{Xe}{ }^{*}=\exp \left\{\mathrm{a}-(\mathrm{b} \cdot \mathrm{T})+\left[\mathrm{c} \cdot \exp \left(\mathrm{a}_{\mathrm{w}}\right)\right]\right\}$ & $0,571297^{* *}$ & $0,011807^{* *}$ & $0,967917^{* *}$ \\
\hline Significativo a 1\% pelo teste t. & &
\end{tabular}

Na Figura 1 estão apresentados os valores experimentais do teor de água de equilíbrio dos frutos de crambe, obtidos por dessorção pelo método dinâmico, bem como suas isotermas estimadas pelo modelo de Sigma Copace.

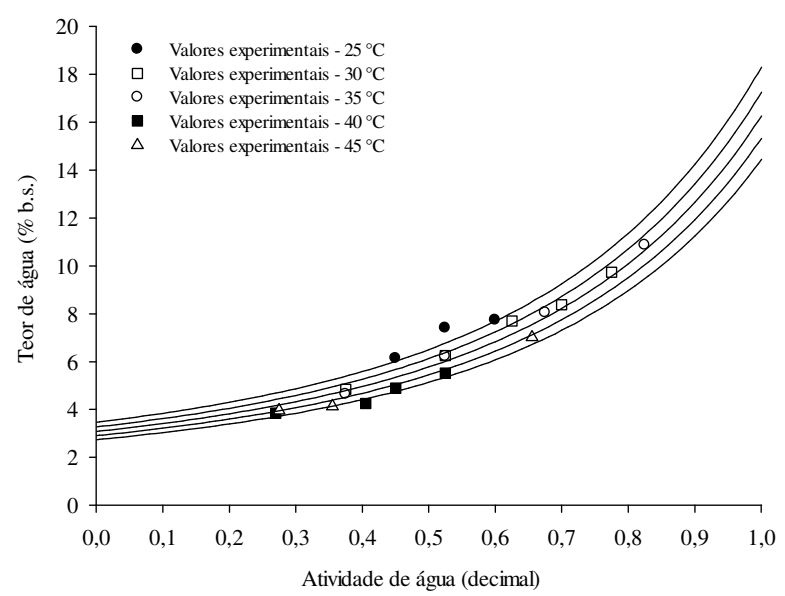

Figura 1. Valores experimentais de teor de água de equilíbrio higroscópico e isotermas de dessorção estimadas pelo modelo de Sigma Copace para os frutos de crambe, em diferentes condições de temperatura e atividades de água.

Observa-se, na Figura 1, que as isotermas de dessorção dos frutos de crambe não se apresentaram na forma típica sigmoidal (tipo II), como observado para a maioria dos produtos agrícolas, como, por exemplo, mamona (GONELI, 2008), soja (AVIRA et al., 2004), trigo (CORRÊA et al., 2005), feijão (RESENDE et al., 2006), grão de bico, sementes de lentilhas, batata e pimentão verde (FURMANIAK et al., 2007), arroz em casca (IGUAZ; VÍRSEDA, 2007) e), sendo classificadas como do tipo 
III(IUPAC, 1985). Park e Nogueira (1992) definem este tipo de isoterma segundo a classificação de BET, quando sua forma tem aparência da letra J. Tal comportamento indica que os principais constituintes do produto (solutos) apresentam pouca afinidade pelas moléculas de água, o que é perfeitamente explicável por ser o produto constituído prioritariamente por lipídios e carboidratos (PENA et al., 2010).

Verifica-se ainda que para uma atividade de água constante os valores de teor de água de equilíbrio higroscópico dos frutos de crambe diminuíram com o aumento da temperatura, seguindo a mesma tendência da maioria dos produtos agrícolas de acordo com Goneli (2008). De um modo geral, a utilização das isotermas de sorção gera informações referentes ao armazenamento adequado e a tomada de decisão sobre medidas preventivas para a manutenção da qualidade do produto. $\mathrm{Na}$ literatura podem ser encontrados diversos trabalhos que relacionam teores iniciais de atividade de água com o desenvolvimento de diversos microrganismos responsáveis pelas sínteses de produtos obtidos em processo de fermentação semi-sólida.

Na Figura 2, são apresentados os valores do calor isostérico integral de dessorção $\left(\mathrm{Q}_{\mathrm{st}}\right)$, em função do teor de água de equilíbrio (\% b.s.), estimados de acordo com a Equação 18.

De acordo com a Figura 2, observa-se que com a redução do teor de água, ocorre aumento da energia necessária para a remoção de água do produto, representada pelos valores do calor isostérico integral de dessorção $\left(\mathrm{Q}_{\mathrm{st}}\right)$. Comportamento semelhante foi observado para diversos produtos agrícolas como batata (ALMUHTASEB et al., 2004); noz pistache (HAYOGLU; GAMLI, 2007); feijão (RESENDE et al., 2006) e quinoa (TOLABA et al., 2004).

*** Significativo a $1 \%$ pelo teste $\mathrm{t}$.



Figura 2. Valores experimentais e estimados do calor isostérico integral de dessorção em função do teor de água de equilíbrio.

Tagawa et al. (1993), afirmam que para retirar água de sementes com baixo teor de água é necessário uma quantidade de energia, em média, superior àquela necessária para produtos úmidos. A proporcionalidade inversa do calor de sorção com o teor de água de equilíbrio está de acordo com o estudo de Iglesias e Chirife (1976), que atribuíram este comportamento ao processo de sorção.

Os valores de calor isostérico integral de dessorção, para os frutos de crambe na faixa de teor de água de 3,85 a 10,86 (\% b.s.) variaram de 4731,73 a $2698,35 \mathrm{~kJ} \mathrm{~kg}^{-1}$. Estes valores divergem aos encontrados por Goneli (2008) que, trabalhando com mamona na faixa de teor de água de 4,77 a 18,91 (\% b.s.), obtiveram valores de calor isostérico integral de dessorção variando de 3324 a 2486 kJ $\mathrm{kg}^{-1}$, aproximadamente.
O elevado valor de calor isostérico integral de sorção para baixos valores de teor de água pode ser explicado pelas diferenças nas forças de ligação da água com a superfície adsorvente de um determinado produto. Em estágios iniciais de sorção, ou seja, com baixos teores de água, existem sítios polares de sorção altamente ativos de elevada energia de interação na superfície adsorvente, que são cobertos com moléculas de água formando uma camada monomolecular (AL-MUHTASEB et al., 2004). À medida que as moléculas de água vão se ligando quimicamente aos sítios de sorção ativos a sorção passa a ocorrer em sítios menos ativo onde estão com elevado teor de água, com menor energia de interação e, consequentemente, menor calor isostérico de sorção (WANG; BRENAN, 1991). 
Determinação das isotermas...

\section{CONCLUSÕES}

O teor de água de equilíbrio higroscópico dos frutos de crambe é diretamente proporcional à atividade de água e decresce com o aumento de temperatura, para um mesmo valor de atividade de água.

Os modelos analisados ajustaram-se bem aos dados experimentais, sendo o modelo de Sigma Copace o que melhor representa os dados experimentais.
COSTA, L. M.; RESENDE, O.; OLIVEIRA, D. E. C.

O calor isostérico aumenta com a diminuição do teor de água de equilíbrio sendo necessária uma maior quantidade de energia para retirar a água dos frutos de crambe.

\section{AGRADECIMENTOS}

Ao Conselho Nacional de Desenvolvimento Científico e Tecnológico (CNPq) e a Coordenação de Aperfeiçoamento de Pessoal de Nível superior (CAPES), pelo apoio financeiro.

\begin{abstract}
The objective of this work was to determine the sorption isotherms of crambe fruits by dynamic method for several conditions of temperature and water activity and adjust different mathematical models to experimental data, and obtain the values of isosteric heat of desorption versus the equilibrium moisture content of the product. The equilibrium moisture content of crambe fruits was determined by dynamic-gravimetric method to temperatures of 25,30 , 35,40 and $45^{\circ} \mathrm{C}$ and water activity for each temperature, from 0.270 to 0.825 . From the results obtained it was calculated for each isosteric heat of moisture content equilibrium. The experimental data were adjusted several models available in the literature (Chung-pfost, Copace, GAB, Modified Halsey, Sabbah, Sigma Copace, Cavalcanti Mata, Modified Henderson, Henderson, BET, Oswin e Modified GAB). It was observed that the equilibrium moisture content decreases with increasing temperature for a given water activity like the hygroscopic products. The model Sigma Copace was best described what the hygroscopicity of the crambe fruits. It was observed that the isosteric heat increases with decreasing moisture content of the product, indicating an increase in energy required for water removal. The values of isosteric heat of crambe fruits in the range of moisture content from 3.85 to 11.86 (d.b.\%) varied from 4731.73 to $2698.35 \mathrm{~kJ} \mathrm{~kg}^{-1}$.
\end{abstract}

KEYWORDS: Crambe abyssinica. Oilseed. Mathematical modeling. Isosteric heat.

\title{
REFERÊNCIAS
}

AL-MUHTASEB, A. H.; MCMINN, W. A. M.; MAGGE, T. R. A. Water sorption isotherms of starch pwders. Part 2: Thermodynamic characteristics. Journal of Food Engineering, Londres, v. 62, n. 2, p. 135-142, 2004. http://dx.doi.org/10.1016/S0260-8774(03)00202-4

AVIRA, N. A.; AJIBOLA, O. O.; ONI, S. A. Sorption equilibrium and thermodynamic characteristics of soya bean. Biosystems Engineering, London, v. 87, n. 2, p. 179-190, 2004.

http://dx.doi.org/10.1016/j.biosystemseng.2003.11.006

BRASIL, Ministério da Agricultura e Reforma Agrária. Secretaria Nacional de defesa Agropecuária. Regras para análise de sementes. Brasília, 2009. 398p.

BROOKER, D. B.; BAKKER-ARKEMA, F. W.; HALL, C. W. Drying and storage of grains and oilseeds. Westport: The AVI Publishing Company, 1992. 450p.

CAMPOS, A. N. R.; SILVA F. L. H.; GOMES, J. P.; OLIVEIRA, L. S. C.; OLIVEIRA, M. M. Isotermas de adsorção e calor isostérico da palma forrageira enriquecida proteicamente. Revista Brasileira de Engenharia Agrícola e Ambiental, Campina Grande, v. 13, n. 6, p. 734-740, 2009.

CORREAA, P. C.; GONELI, A. L. D.; RESENDE, O.; RIBEIRO, D. M. Obtenção e modelagem das isotermas de dessorção e do calor esotérico de dessorção para grãos de trigo. Revista Brasileira de Produtos Agroindustriais, Campina Grande, v. 7, n. 1, p. 39-48, 2005.

FERREIRA, C. D.; PENA, R. S. Comportamento higroscópico da farinha de pupunha (Bactris gasipaes).

Ciência e Tecnologia de Alimentos, Campinas, v. 23, n. 2, p. 251-255, 2003. http://dx.doi.org/10.1590/S010120612003000200025 
FURMANIAK, S.; TERZYK, A. P.; GAUDEN, P. A. The general mechanism of water sorption on foodstuffs Importance of the multitemperature fitting of data and the hierarchy of models. Journal of Food Engineering, Londres, v. 82, n. 4, p. 528-535, 2007.

GONELI, A. L. D. Variação das propriedades físicas-mecânicas e da qualidade da mamona (Ricinus communis L.) durante a secagem e o armazenamento. Viçosa, 2008. 186 f. Tese (Doutorado em Engenharia Agrícola) - Universidade Federal de Viçosa, Viçosa.

HAYOGLU, I.; GAMLI, O. F. Water sorption isotherms of pistachio nut paste. International Journal of Food Science and Technology, Mysore, v. 42, n. 2, p. 224-227. 2007. http://dx.doi.org/10.1111/j.1365-

2621.2006.01283.x

HOFSKY, A. V.; FIGUEIREDO, R. M. F.; QUEIROZ, A. J. M. Isotermas de adsorção de umidade da pitanga em pó. Revista de Biologia e Ciências da Terra, Paraíba, v. 7, n. 1, p. 11-20, 2007.

IGLESIAS, H.; CHIRIFE, J. Prediction of the effect of temperature on water sorption isotherms of food material. Journal of Food Technology, Oxford, v. 11, n. 2, p. 109-116, 1976. http://dx.doi.org/10.1111/j.13652621.1976.tb00707.x

IGUAZ, A.; VÍRSEDA, P. Moisture desorption isotherms of roung rice at high temperatures. Journal of Food Engineering, Londres, v. 79, n. 3, p. 794-802, 2007. http://dx.doi.org/10.1016/j.jfoodeng.2006.03.002

IUPAC - International Union of Pure and Applied Chemistry. Reporting physisorption data for gas/solid systems. Pure and Applied Chemistry. Oxford , v. 57, n. 4, p. 603-619, 1985.

KNIGHTS S. E. Crambe A North Dakota Case Study. Rural Industries Research and Development Corporation, RIRDC Publication No W02/005, RIRDC Project No TA001-55, fev. 2002. Disponível em: http://www.rirdc.gov.au/reports/NPP/02-005.pdf. Acesso em 16 nov. 2008.

MADAMBA, P. S.; DRISCOLL, R. H.; BUCKLE, K. A. Thin-layer drying characteristics of garlic slices. Journal of Food Engineering, Londres, v. 29, n. 1, p. 75-97,1996. http://dx.doi.org/10.1016/02608774(95)00062-3

MELLO, M. G. Biomassa. Energia dos trópicos em Minas Gerais. Editora Labmídia. Belo Horizonte, 2011.

MESQUITA, J. B.; ANDRADE, E. T.; CORRÊA, P. C. Modelos matemáticos e curvas de umidade de equilíbrio de sementes de jacarandá-da-bahia, angico-vermelho e óleo-copaíba. Cerne, Lavras, v. 7, n. 2, p. 12$21,2001$.

MOHAPATRA, D.; RAO, P. S. A thin layer drying model of parboiled wheat. Journal of Food Engineering, Londres, v. 66, n. 4, p. 513-518, 2005. http://dx.doi.org/10.1016/j.jfoodeng.2004.04.023

MOREIRA, M. F. P. Secagem de Gel Agar. Dissertação (Mestrado em Engenharia Química). São Paulo: Universidade Federal de São Carlos, 2000.

NELLIST, M. E.; HUGUES, M. Physical and biological processes in the drying of seed. Seed Science and Technology, Zürich, v. 1, n. 3 p. 613-643, 1973.

OPLINGER, E. S. et al. Crambe. Alternative Field Crops Manual. Disponível em: $<$ http://www.hort.purdue.edu/newcrop/afcm/crambe.html>. Acesso em 16 nov. 2008.

PARK, K. J.; NOGUEIRA, R. I. Modelo para ajuste de isoterma de sorção de alimentos. Revista Engenharia Rural, Piracicaba. 3, v. 1, p. 80-86, 1992. 
PENA, R. S.; MENDONÇA, N. B.; ALMEIDA, M. D. C. Comportamento higroscópico do açaí em pó. Revista Brasileira de Produtos Agroindustriais, Campina Grande, v. 12, n. 2, p. 153-161, 2010.

RESENDE, O.; CORREAA, P. C; GONELI, A. L. D.; RIBEIRO, D. M. Isotermas e calor isostérico de sorção do feijão. Revista Ciência e Tecnologia de Alimentos, Campinas, v. 26, n. 3, p. 626-631, 2006.

http://dx.doi.org/10.1590/S0101-20612006000300022

SOPADE, P. A.; AJISEGIRI, E. S. Moisture sorption study on nigerian foods: Maize and sorghum. Journal of Food Process Engineering, Lleidav, 17, n. 1, p. 33-56, 1994.

SOUSA, K. A.; RESENDE, O.; COSTA, L. M. Isotermas de dessorção das sementes de nabo forrageiro obtidas pelos métodos dinâmico e estático. Revista Brasileira de Engenharia Agrícola e Ambiental, Campina Grande, v. 17, n. 2, p. 216-222, 2013.

TAGAWA, A.; MURATA, S.; HAYASHI, H. Latent heat of vaporization in buckwheat using the data of equilibrium moisture content. Transactions of the ASAE, St. Joseph, v. 36, n. 1, p. 113-118, 1993.

TOLABA, M. P.; PELTZER, M.; ENRIQUEZ, N.; POLLIO, M. L. Grain sorption equilibria of quinoa grains. Journal of Food Engineering, Londres, v. 61, n. 3, p. 365-371, 2004. http://dx.doi.org/10.1016/S02608774(03)00143-2

WANG, N.; BRENNAN, J. G. Moisture sorption isotherm characteristics of potato at four temperatures. Journal of Food Engineering, Londres, v. 14, n. 4, p. 269-287, 1991. http://dx.doi.org/10.1016/02608774(91)90018-N 